Darío Correa-Restrepo and Dieter Pfirsch

The electromagnetic gauge in the variational formulation of kinetic and other theories

IPP $5 / 107$

June 2004

Submitted to J. Plasma Phys. 2004. 


\title{
The electromagnetic gauge in the variational formulation of kinetic and other theories
}

\author{
Darío Correa-Restrepo and Dieter Pfirsch \\ Max-Planck-Institut für Plasmaphysik, EURATOM Association, D-85748 Garching, Germany \\ (dcr@ipp.mpg.de)
}

\begin{abstract}
This paper investigates the implications and consequences of choosing special gauges or gauge-invariant or non-gauge-invariant approximations in the action integral for the variational formulation of theories involving electromagnetic fields. After some interesting special gauges are considered, it is shown that non-gauge-invariant approximations always lead to inconsistent Euler-Lagrange equations. As a concrete example, the Maxwell-Vlasov theory is investigated. The special non-gauge-invariant case considered, which is sometimes used in the literature, is obtained by replacing the contribution of the electric field to the Maxwell part of the Lagrangian density by the contribution of the gradient of the scalar potential alone. The detailed investigation concerns the local energy conservation law, and it is shown that the law thus derived is physically meaningless since it contains non-physical, spurious terms. The results are also valid for drift-kinetic and gyrokinetic theories, and for other theories, e.g. two-fluid theories.
\end{abstract}




\section{Contents}

1 Introduction 3

2 Special gauges used in the exact Maxwell Lagrangian density 4

3 Non-gauge-invariant versus gauge-invariant approximations of the Maxwell Lagrangian density

4 Detailed investigation of the deficiencies resulting from different non-gauge-invariant approximations of the Maxwell La$\begin{array}{ll}\text { grangian density within Maxwell-Vlasov theory } & 7\end{array}$

4.1 Variation of the Lagrangian density . . . . . . . . . . . . 9 9

4.2 Euler-Lagrange equations . . . . . . . . . . . . . 10

4.2.1 Equation for the potentials containing the charge density 10

4.2.2 Equation for the potentials containing the current density 10

4.2.3 Hamilton-Jacobi equations . . . . . . . . . . . 10

4.2 .4 Kinetic equations . . . . . . . . . . . . 10

4.3 Local energy conservation . . . . . . . . . . . . . 11

4.3.1 Gauge-invariant $\mathcal{L}_{\mathrm{M}}=(1 / 8 \pi)\left[|\boldsymbol{\nabla} \Phi+(1 / c) \partial \mathbf{A} / \partial t|^{2}-\mathbf{B}^{2}\right] \quad 14$

4.3.2 Non-gauge-invariant approximation with $\mathcal{L}_{\mathrm{M}}=(1 / 8 \pi)\left[|\boldsymbol{\nabla} \Phi|^{2}-\mathbf{B}^{2}\right] \ldots \ldots \ldots . . \ldots 14$

4.3.3 Difference of neglecting $(1 / c) \partial \mathbf{A} / \partial t$ in $\mathcal{L}_{\mathrm{M}}$ or in final results 16

4.3.4 Gauge-invariant approximation with $\mathcal{L}_{\mathrm{M}}=(1 / 8 \pi)\left[|\boldsymbol{\nabla} \Phi+(1 / c) \partial \mathbf{A} / \partial t|^{2}\right] \ldots \ldots \ldots$

4.3.5 Gauge-invariant approximation with $\mathcal{L}_{\mathrm{M}}=-(1 / 8 \pi) \mathbf{B}^{2} \quad .17$ 


\section{Introduction}

The electromagnetic potentials $\Phi$ and $\mathbf{A}$ are related to the fields $\mathbf{E}$ and $\mathbf{B}$ by the equations

$$
\mathbf{E}=-\nabla \Phi-\frac{1}{c} \frac{\partial \mathbf{A}}{\partial t} \text { and } \mathbf{B}=\boldsymbol{\nabla} \times \mathbf{A} .
$$

The electric and magnetic fields are not modified when the potentials are changed according to gauge transformations

$$
\Phi^{\prime}=\Phi-\frac{1}{c} \frac{\partial \Psi}{\partial t}, \quad \mathbf{A}^{\prime}=\mathbf{A}+\nabla \Psi
$$

A theory defined by a variational problem is gauge invariant if the action integral is invariant under such gauge transformations or if the Lagrangian density only changes by a time derivative. It is then obvious that all results concerning physical quantities obtained from Hamilton's principle do not depend on the gauge.

On the other hand, if a theory is not gauge invariant the results have, in principle, no physical meaning, since changing the gauge changes the results. It is, of course, allowed to use a special gauge with all the results being physically meaningful, but Hamilton's principle in this case must be supplemented by corresponding constraints. However, these constraints must not be combined with non-gauge-invariant approximations of the Maxwell fields, where the gauge transformations are restricted to leave the constraint invariant.

Usually, one considers kinetic theories with gauge-invariant particle contributions to the action integral which yield gauge-invariant charge and current densities satisfying the corresponding continuity equation. But these particle contributions are sometimes combined with Maxwell contributions containing non-gauge-invariant approximations. An example will be considered in Sec. 4.3.2. One problem which immediately arises in this case is the appearance of an inconsistency within the local conservation law for the electric charge, which is one of the possible reasons for the appearance of incorrect, spurious terms in the local energy conservation law. However, even if local charge conservation is formally guaranteed by the introduction of an appropriate Lagrange multiplier term, the expression for the current density remains, nevertheless, incomplete and this, in turn, is responsible for the appearance of a different spurious term in the local energy conservation law. This deficiency does not occur if the approximations are gauge invariant. The first feature will already be shown here in the introduction. Later we will consider the interesting particular example of the Maxwell-Vlasov theory in detail. It will be seen that the incorrect terms concern only the energy flux density; therefore, they do not influence the total energy conservation. However, the dynamics behind this total energy conservation law, which is reflected by the energy flux density, is wrong. The results obtained are typical also of drift-kinetic and gyrokinetic theories, and other theories, e.g. two-fluid theories. 
As concerns the first feature, let the Maxwell contribution to the Lagrangian density be any function of $\mathbf{E}$ and $\mathbf{B}$ :

$$
\mathcal{L}_{\mathrm{M}}=\mathcal{L}_{\mathrm{M}}(\mathbf{E}, \mathbf{B})=\mathcal{L}_{\mathrm{M}}\left(\mathbf{E}=-\boldsymbol{\nabla} \Phi-\frac{1}{c} \frac{\partial \mathbf{A}}{\partial t}, \mathbf{B}=\boldsymbol{\nabla} \times \mathbf{A}\right) .
$$

This is, of course, always gauge invariant. Variation of the total action integral with respect to $\Phi$ and $\mathbf{A}$ yields

$$
\boldsymbol{\nabla} \cdot \frac{\partial \mathcal{L}_{\mathrm{M}}}{\partial \boldsymbol{\nabla} \Phi}=-\boldsymbol{\nabla} \cdot \frac{\partial \mathcal{L}_{\mathrm{M}}}{\partial \mathbf{E}}=-\rho
$$

and

$$
\boldsymbol{\nabla} \times \frac{\partial \mathcal{L}_{\mathrm{M}}}{\partial \mathbf{B}}=-\frac{1}{c} \mathbf{j}+\frac{1}{c} \frac{\partial}{\partial t} \frac{\partial \mathcal{L}_{\mathrm{M}}}{\partial[(1 / c)(\partial \mathbf{A} / \partial t)]}=-\frac{1}{c} \mathbf{j}-\frac{1}{c} \frac{\partial}{\partial t} \frac{\partial \mathcal{L}_{\mathrm{M}}}{\partial \mathbf{E}},
$$

which is obviously in agreement with

$$
\frac{\partial \rho}{\partial t}+\nabla \cdot \mathbf{j}=0
$$

for all choices of $\mathcal{L}_{\mathrm{M}}(\mathbf{E}, \mathbf{B})$ representing certain approximations. Similarly, one can show that all Maxwell Lagrangian densities of the form $\mathcal{L}_{\mathrm{M}}(\boldsymbol{\nabla} \Phi, \partial \mathbf{A} / \partial t, \boldsymbol{\nabla} \times$ A) $\neq \mathcal{L}_{\mathrm{M}}(\mathbf{E}, \mathbf{B})$ lead to inconsistencies.

In Section 2 we consider three examples with prescribed gauges. Section 3 treats an example with a non-gauge-invariant choice for $\mathcal{L}_{\mathrm{M}}$, which is later studied in detail in Section 4, and, in particular, in Section 4.3.2. Two further examples with prescribed gauge-invariant $\mathcal{L}_{\mathrm{M}}(\mathbf{E}, \mathbf{B})$ are also treated in Section 3 ; they correspond to linearized theories with purely electrostatic perturbations and to quasi-neutral theories, respectively. They are proposed as a way of replacing non-gauge-invariant expressions for $\mathcal{L}_{\mathrm{M}}$. These last two examples are again treated in Section 4, and, in particular, in Sections 4.3.4 and 4.3.5, respectively.

\section{Special gauges used in the exact Maxwell Lagrangian density}

In the investigation of kinetic plasma theories it is sometimes convenient to introduce a special gauge for the electromagnetic potentials $\Phi$ and $\mathbf{A}$ in the exact Maxwell Lagrangian density,

$$
\mathcal{L}_{\mathrm{M}}=\frac{1}{8 \pi}\left[\mathbf{E}^{2}-\mathbf{B}^{2}\right]=\frac{1}{8 \pi}\left[\left|\boldsymbol{\nabla} \Phi+\frac{1}{c} \frac{\partial \mathbf{A}}{\partial t}\right|^{2}-\mathbf{B}^{2}\right] .
$$

Interesting examples of exact gauges are

$$
\boldsymbol{\nabla} \cdot \mathbf{A}=0, \quad \frac{1}{c} \frac{\partial \Phi}{\partial t}+\boldsymbol{\nabla} \cdot \mathbf{A}=0, \quad \Phi=0 .
$$


The first one is the Coulomb gauge, the second one is the Lorentz gauge, and the third one is obtained from any special gauge by a gauge transformation given by

$$
\mathbf{A}=\mathbf{A}^{\prime}+\nabla \Psi, \quad \Phi=\Phi^{\prime}-\frac{1}{c} \frac{\partial \Psi}{\partial t}, \quad \text { with } \frac{\partial \Psi}{\partial t}=c \Phi^{\prime} .
$$

When any of these gauges is used in the variational problem, the allowed variations are restricted. One can take care of this by introducing Lagrange multipliers.

The Coulomb gauge does not change the Lagrangian density. Therefore, only the Euler-Lagrange equations are modified. Because of the term

$$
\mu_{\mathrm{C}} \boldsymbol{\nabla} \cdot \mathbf{A}
$$

relating to the Lagrange multiplier $\mu_{\mathrm{C}}$, there is a contribution to the right-hand side of the equation for $\boldsymbol{\nabla} \times \mathbf{B}$, given by

$$
\nabla \mu_{\mathrm{C}}
$$

However, the equation for $\boldsymbol{\nabla} \times \mathbf{B}$ must remain unchanged. Hence,

$$
\nabla \mu_{\mathrm{C}}=0
$$

In the case of the Lorentz gauge, one has analogously the additional expression

$$
\mu_{\mathrm{L}}\left[\frac{1}{c} \frac{\partial \Phi}{\partial t}+\nabla \cdot \mathbf{A}\right],
$$

from which the additional charge density

$$
\rho_{\text {add }}=-\frac{1}{c} \frac{\partial \mu_{\mathrm{L}}}{\partial t}
$$

follows. This must vanish and, therefore,

$$
\frac{\partial \mu_{\mathrm{L}}}{\partial t}=0
$$

There is also an additional current density

$$
\mathbf{j}_{\mathrm{add}}=-\nabla \mu_{\mathrm{L}}
$$

which must also vanish. Hence, also

$$
\nabla \mu_{\mathrm{L}}=0
$$

In the case of the gauge $\Phi=0$, there is the additional expression

$$
\mu_{\Phi} \Phi
$$


relating to the Lagrange multiplier $\mu_{\Phi}$. This yields an additional charge density

$$
\rho_{\text {add }}=\mu_{\phi}
$$

On the other hand, since in this case

$$
\mathbf{E}=-\frac{1}{c} \frac{\partial \mathbf{A}}{\partial t}
$$

there is now no term $\boldsymbol{\nabla} \cdot \mathbf{E}$ resulting from the $\mathbf{E}^{2}$ contribution to $\mathcal{L}_{\mathrm{M}}$. Therefore, one must choose

$$
4 \pi \mu_{\Phi}=\nabla \cdot \mathbf{E}
$$

in order to obtain the correct Maxwell equations.

These choices of the various Lagrange multipliers, together with Maxwell's equations, leave all conservation laws unchanged.

\section{Non-gauge-invariant versus gauge-invariant approximations of the Maxwell Lagrangian density}

The situation is different with non-gauge-invariant Maxwell Lagrange densities obtained from certain approximations. An example is the non-gauge-invariant choice

$$
\mathcal{L}_{\mathrm{M}}=\frac{1}{8 \pi}\left[|\boldsymbol{\nabla} \Phi|^{2}-(\boldsymbol{\nabla} \times \mathbf{A})^{2}\right] .
$$

The usual argument for this choice is that one is interested in situations where the condition

$$
\left|\frac{1}{c} \frac{\partial \mathbf{A}}{\partial t}\right| \ll|\nabla \Phi| \mid
$$

is satisfied. There is, of course, no exact gauge such that $\partial \mathbf{A} / \partial t=0$, contrary to $\nabla \Phi=0$. Moreover, it must be stressed that within the variational problem one does not compare the contributions to the factors of $\delta \Phi$ with the contributions to the factors of $\delta \mathbf{A}$. The comparison is made separately within the factor of $\delta \Phi$ and within the factor of $\delta \mathbf{A}$. If $(1 / c) \partial \mathbf{A} / \partial t$ is already neglected in the Lagrangian density, incorrect and inconsistent Euler-Lagrange equations are obtained, not only in the sense discussed in the Introduction, but also inconsistent in the assumed approximation (23) itself. This will be explained in detail further below in Sec. 4 . It can be illustrated by means of, for example, (46), where it is readily seen that the contribution $(1 / 4 \pi) \partial \nabla \Phi / \partial t$ to the displacement current density gets lost if the term $(1 / c) \partial \mathbf{A} / \partial t$ is already neglected in the Lagrangian density $\mathcal{L}_{\mathrm{M}}$, with the consequence that one obtains not only incorrect and inconsistent Euler-Lagrange equations, but also wrong expressions for the conservation laws. The fundamental inconsistency results 
from the variation of the action integral, with (22) taken into account, with respect to $\Phi$ and $\mathbf{A}$. This obviously leads to the following two inhomogeneous Maxwell equations:

$$
\Delta \Phi=-4 \pi \rho \text { and } \nabla \times \mathbf{B}=\frac{4 \pi}{c} \mathbf{j}
$$

where $\rho$ and $\mathbf{j}$ are calculated, e.g. from the Vlasov distribution functions, and are time dependent. Hence, (24) imply the inconsistency

$$
\frac{\partial \rho}{\partial t} \neq 0 \text { and } \boldsymbol{\nabla} \cdot \mathbf{j}=0
$$

It could be argued that one is essentially interested in quasi-neutral situations with $\partial \rho / \partial t$ negligible. In this case, however, one should choose for $\mathcal{L}_{\mathrm{M}}$ the gauge-invariant expression

$$
\mathcal{L}_{\mathrm{M}}=-\frac{1}{8 \pi} \mathbf{B}^{2}
$$

which directly yields the exact quasi-neutral equations

$$
\rho=0 \text { and } \boldsymbol{\nabla} \times \mathbf{B}=\frac{4 \pi}{c} \mathbf{j} .
$$

This implies a Poynting vector with

$$
\mathbf{E}=-\nabla \Phi-\frac{1}{c} \frac{\partial \mathbf{A}}{\partial t}
$$

but the energy density consists only of the magnetic field density. This case is further discussed in Sec. 4.3.5.

Another argument could be that one is interested only in electrostatic perturbations within a linearized theory. In this case one should replace

$$
\mathcal{L}_{\mathrm{M}}^{(2)}=\frac{1}{8 \pi}\left[\left(\mathbf{E}^{(1)}\right)^{2}-\left(\mathbf{B}^{(1)}\right)^{2}\right] \rightarrow \frac{1}{8 \pi}\left(\mathbf{E}^{(1)}\right)^{2},
$$

but still with

$$
\mathbf{E}^{(1)}=-\nabla \Phi^{(1)}-\frac{1}{c} \frac{\partial \mathbf{A}^{(1)}}{\partial t}
$$

which again means a gauge-invariant theory. There is then no longer a Poynting vector, and the second-order energy density only contains an electric field energy. This question is treated, basically, in Sec. 4.3.4.

\section{Detailed investigation of the deficiencies re- sulting from different non-gauge-invariant ap- proximations of the Maxwell Lagrangian den- sity within Maxwell-Vlasov theory}

The Maxwell-Vlasov theory is studied here with techniques which are basically those of [1] and references therein, using variational methods. To simplify 
matters, the method is again briefly given here. The action functional for the theory is

$$
\mathcal{A}=\int d t d^{3} x \mathcal{L}_{\text {tot. }}
$$

where

$$
\mathcal{L}_{\text {tot. }}=\mathcal{L}_{\mathrm{M}}+\mathcal{L}_{\mathrm{K}}
$$

is the total Lagrangian density consisting of Maxwell field and kinetic contributions, $\mathcal{L}_{\mathrm{M}}$ and $\mathcal{L}_{\mathrm{K}}$, respectively. Here we are only interested in deriving the local energy conservation law and, in particular, comparing different theories characterized by different approximations of the Maxwell Lagrangian density. This density, in the general case, is given by

$$
\mathcal{L}_{\mathrm{M}}(\mathbf{x}, t)=\frac{1}{8 \pi}\left(\mathbf{E}^{2}-\mathbf{B}^{2}\right)=\frac{1}{8 \pi}[|\overbrace{\boldsymbol{\nabla} \Phi}^{\mathrm{E} 1}+\overbrace{\frac{1}{c} \frac{\partial \mathbf{A}}{\partial t}}^{\mathrm{E} 2}|^{2}-\overbrace{\mathbf{B}^{2}}^{\mathrm{M}}] .
$$

The overbraces characterizing the different terms are introduced here for easy description of the different cases treated below, and are very convenient in tracking the contribution of the different terms to the local energy conservation law. Later, terms with the characterization E1,E2 above the overbrace will be encountered. Such terms vanish when either $\boldsymbol{\nabla} \Phi$ or $(1 / c) \partial \mathbf{A} / \partial t$ or both vanish in the Maxwell Lagrangian density.

The kinetic Lagrangian density is given by

$$
\mathcal{L}_{\mathrm{K}}(\mathbf{x}, t)=-\sum_{\mathrm{p.s.}} \int d^{3} \alpha f_{\mathrm{p}}(\mathbf{x}, \boldsymbol{\alpha}, t)\left[\frac{\partial S_{n}(\mathbf{x}, \boldsymbol{\alpha}, t)}{\partial t}+H\left(\mathbf{x}, \frac{\partial S_{n}}{\partial \mathbf{x}}, t\right)\right],
$$

where $H(\mathbf{x}, \mathbf{p}, t)=e \Phi(\mathbf{x}, t)+\hat{H}(\mathbf{p}-(e / c) \mathbf{A}(\mathbf{x}, t))$ is the particle Hamiltonian, with $\hat{H}$ given by

$$
\hat{H}(\mathbf{x}, \mathbf{p}, t)=\frac{1}{2 m}\left[\mathbf{p}-\frac{e}{c} \mathbf{A}(\mathbf{x}, t)\right]^{2} .
$$

As shown below, the functions $S(\mathbf{x}, \boldsymbol{\alpha}, t)$ satisfy the Hamilton-Jacobi equations, $\partial S / \partial t+H(\mathbf{x}, \partial S / \partial \mathbf{x}, t)=0$, as a result of the variational principle. These functions are only used as a tool. They will not appear in the final expressions and, therefore, one does not have to solve the Hamilton-Jacobi equations; only certain general properties of the functions $S(\mathbf{x}, \boldsymbol{\alpha}, t)$ will play a role. The $\boldsymbol{\alpha}$ 's are constants of integration for the Hamilton-Jacobi equations. $S(\mathbf{x}, \boldsymbol{\alpha}, t)$ is a mixed-variable generating function for a canonical transformation to new Hamiltonians equal to zero. The new momenta $\boldsymbol{\alpha}$ and coordinates $\boldsymbol{\beta}=\partial S(\mathbf{x}, \boldsymbol{\alpha}, t) / \partial \boldsymbol{\alpha}$ are therefore constants of motion. The functions $f_{\mathrm{p}}(\mathbf{x}, \boldsymbol{\alpha}, t)$ are primary forms of the distribution functions with the argument $\boldsymbol{\alpha}$ instead of $\mathbf{p}$ or $\mathbf{v}$. The quantities to be varied in Hamilton's principle are, besides $\Phi$ and $\mathbf{A}$, the functions $S(\mathbf{x}, \boldsymbol{\alpha}, t)$ and $f_{\mathrm{p}}(\mathbf{x}, \boldsymbol{\alpha}, t)$. The gauge transformation

$$
\Phi \rightarrow \Phi-\frac{1}{c} \frac{\partial \Psi}{\partial t}, \quad \mathbf{A} \rightarrow \mathbf{A}+\nabla \Psi, \quad S \rightarrow S+\frac{e}{c} \Psi
$$


leaves both contributions to the total Lagrangian density invariant when the electric field considered includes both $\boldsymbol{\nabla} \Phi$ and $(1 / c) \partial \mathbf{A} / \partial t$.

\subsection{Variation of the Lagrangian density}

The variation of the Lagrangian density is

$$
\delta \mathcal{L}_{\text {tot. }}=\delta \mathcal{L}_{\mathrm{K}}+\delta \mathcal{L}_{\mathrm{M}}
$$

The variation of the kinetic Lagrangian density can be written as

$$
\begin{aligned}
\delta \mathcal{L}_{\mathrm{K}}(\mathbf{x}, t)= & \delta_{\delta \Phi} \mathcal{L}_{\mathrm{K}}(\mathbf{x}, t)+\delta_{\delta \mathbf{A}} \mathcal{L}_{\mathrm{K}}(\mathbf{x}, t) \\
& +\delta_{\delta f_{\mathrm{p}}} \mathcal{L}_{\mathrm{K}}(\mathbf{x}, t)+\delta_{\delta S} \mathcal{L}_{\mathrm{K}}(\mathbf{x}, t),
\end{aligned}
$$

with

$$
\begin{gathered}
\delta_{\delta \Phi} \mathcal{L}_{\mathrm{K}}(\mathbf{x}, t)=-\sum_{\text {p. s. }} \int d^{3} \alpha f_{\mathrm{p}}(\mathbf{x}, \boldsymbol{\alpha}, t) e \delta \Phi(\mathbf{x}, t) \\
\delta_{\delta \mathbf{A}} \mathcal{L}_{\mathrm{K}}(\mathbf{x}, t)=-\sum_{\text {p. s. }} \int d^{3} \alpha f_{\mathrm{p}}(\mathbf{x}, \boldsymbol{\alpha}, t)\left[-\frac{e}{c} \delta \mathbf{A}(\mathbf{x}, t)\right] \cdot \frac{\partial H}{\partial \mathbf{p}}, \\
\delta_{\delta f_{\mathrm{p}}} \mathcal{L}_{\mathrm{K}}(\mathbf{x}, t)=-\sum_{\text {p. s. }} \int d^{3} \alpha \delta f_{\mathrm{p}}(\mathbf{x}, \boldsymbol{\alpha}, t)\left[\frac{\partial S(\mathbf{x}, \boldsymbol{\alpha}, t)}{\partial t}+H\right]
\end{gathered}
$$

and

$$
\begin{aligned}
\delta_{\delta S} \mathcal{L}_{\mathrm{K}}(\mathbf{x}, t)= & -\sum_{\mathbf{p} . \mathrm{s.}} \int d^{3} \alpha f_{\mathrm{p}}(\mathbf{x}, \boldsymbol{\alpha}, t)\left[\frac{\partial \delta S(\mathbf{x}, \boldsymbol{\alpha}, t)}{\partial t}+\frac{\partial \delta S}{\partial \mathbf{x}} \cdot \frac{\partial H}{\partial \mathbf{p}}\right] \\
= & -\sum_{\mathbf{p} . \mathrm{s} .} \int d^{3} \alpha\left[-\delta S(\mathbf{x}, \boldsymbol{\alpha}, t)\left[\frac{\partial f_{\mathrm{p}}}{\partial t}+\frac{\partial}{\partial \mathbf{x}} \cdot\left(f_{\mathrm{p}} \frac{\partial H}{\partial \mathbf{p}}\right)\right]\right. \\
& \left.+\frac{\partial}{\partial t}\left(f_{\mathrm{p}} \delta S\right)+\frac{\partial}{\partial \mathbf{x}} \cdot\left(f_{\mathrm{p}} \delta S \frac{\partial H}{\partial \mathbf{p}}\right)\right]
\end{aligned}
$$

where

$$
\frac{\partial H}{\partial \mathbf{p}}=\left.\frac{\partial H(\mathbf{x}, \mathbf{p}, t)}{\partial \mathbf{p}}\right|_{\mathbf{p}=\partial S(\mathbf{x}, \boldsymbol{\alpha}, t) / \partial \mathbf{x}}=\mathbf{v}\left(\mathbf{x}, \mathbf{p}=\frac{\partial S}{\partial \mathbf{x}}, t\right) .
$$

The variation of the Maxwell Lagrangian density is

$$
\begin{aligned}
& \delta \mathcal{L}_{\mathrm{M}}(\mathbf{x}, t) \quad \frac{1}{4 \pi}\{\boldsymbol{\nabla} \cdot[\overbrace{\delta \Phi}^{\mathrm{E} 1}(\overbrace{\boldsymbol{\nabla} \Phi}^{\mathrm{E} 1}+\overbrace{\frac{1}{c} \frac{\partial \mathbf{A}}{\partial t}}^{\mathrm{E} 2})]-\overbrace{\delta \Phi}^{\mathrm{E} 1}(\overbrace{\Delta \Phi}^{\mathrm{E} 1}+\overbrace{\frac{1}{c} \frac{\partial \boldsymbol{\nabla} \cdot \mathbf{A}}{\partial t}}^{\mathrm{E} 2}) \\
& +\frac{\partial}{\partial t}[\overbrace{\frac{\delta \mathbf{A}}{c}}^{\mathrm{E} 2} \cdot(\overbrace{\nabla \Phi}^{\mathrm{E} 1}+\overbrace{\frac{1}{c} \frac{\partial \mathbf{A}}{\partial t}}^{\mathrm{E} 2})]-\overbrace{\frac{\delta \mathbf{A}}{c}}^{\mathrm{E} 2} \cdot \frac{\partial}{\partial t}(\overbrace{\boldsymbol{\nabla} \Phi}^{\mathrm{E} 1}+\overbrace{\frac{1}{c} \frac{\partial \mathbf{A}}{\partial t}}^{\mathrm{E} 2}) \\
& -\overbrace{\delta \mathbf{A}}^{\mathrm{M}} \cdot \overbrace{\boldsymbol{\nabla} \times \mathbf{B}}^{\mathrm{M}}-\nabla \cdot(\overbrace{\delta \mathbf{A}}^{\mathrm{M}} \times \overbrace{\mathbf{B}}^{\mathrm{M}})\} .
\end{aligned}
$$




\subsection{Euler-Lagrange equations}

The use of (37)-(44) in Hamilton's principle yields the electric charge density from the variation $\delta \Phi$, the current density from $\delta \mathbf{A}$, the Hamilton-Jacobi equations from $\delta f_{\mathrm{p}}$ and the Vlasov equations in the variables $\mathbf{x}, \boldsymbol{\alpha}, t$ from $\delta S$.

\subsubsection{Equation for the potentials containing the charge density}

$$
\frac{1}{4 \pi} \overbrace{\Delta \Phi}^{\mathrm{E} 1}+\frac{1}{4 \pi c} \overbrace{\frac{\partial \boldsymbol{\nabla} \cdot \mathbf{A}}{\partial t}}^{\mathrm{E} 1, \mathrm{E} 2}=-\sum_{\mathrm{p} . \mathrm{s} .} e \int d^{3} \alpha f_{\mathrm{p}}(\mathbf{x}, \boldsymbol{\alpha}, t)=-\rho .
$$

As previously pointed out, the terms characterized by E1, E2 vanish when the terms $\mathrm{E} 1$ or E2 or both are omitted in the Lagrangian density.

\subsubsection{Equation for the potentials containing the current density}

$$
\frac{c}{4 \pi} \boldsymbol{\nabla} \times \overbrace{\mathbf{B}}^{\mathrm{M}}+\frac{1}{4 \pi} \frac{\partial}{\partial t}(\overbrace{\nabla \Phi}^{\mathrm{E} 1, \mathrm{E} 2}+\frac{1}{c} \frac{\overbrace{\frac{\partial \mathbf{A}}{\partial t}}^{\mathrm{E} 2}}{\partial t}=\sum_{\mathrm{p} . \mathbf{s} .} e \int d^{3} \alpha f_{\mathrm{p}}(\mathbf{x}, \boldsymbol{\alpha}, t) \frac{\partial H}{\partial \mathbf{p}}=\mathbf{j},
$$

from which

$$
\boldsymbol{\nabla} \cdot \mathbf{j}=\frac{1}{4 \pi} \frac{\partial}{\partial t}[\overbrace{\Delta \Phi}^{\mathrm{E} 1, \mathrm{E} 2}+\frac{1}{c} \overbrace{\frac{\partial \boldsymbol{\nabla} \cdot \mathbf{A}}{\partial t}}^{\mathrm{E} 2}]
$$

follows.

\subsubsection{Hamilton-Jacobi equations}

$$
\frac{\partial S(\mathbf{x}, \boldsymbol{\alpha}, t)}{\partial t}+H=0
$$

\subsubsection{Kinetic equations}

$$
\frac{\partial f_{\mathrm{p}}}{\partial t}+\frac{\partial}{\partial \mathbf{x}} \cdot\left(f_{\mathrm{p}} \frac{\partial H}{\partial \mathbf{p}}\right)=0
$$

After taking the Euler-Lagrange equations into account, the variation $\delta \mathcal{L}_{\text {tot. }}$ reduces to $\delta^{\mathrm{EL}} \mathcal{L}_{\text {tot. }}$, where the superscript EL means that the Euler-Lagrange equations were used.

$$
\begin{aligned}
& \delta^{\mathrm{EL}} \mathcal{L}_{\text {tot. }}(\mathbf{x}, t)=\delta^{\mathrm{EL}}\left(\mathcal{L}_{\mathrm{K}}+\mathcal{L}_{\mathrm{M}}\right)= \\
& -\sum_{\text {p. s. }} \int d^{3} \alpha\left[\frac{\partial}{\partial t}\left(f_{\mathrm{p}} \delta S\right)+\frac{\partial}{\partial \mathbf{x}} \cdot\left(f_{\mathrm{p}} \delta S \frac{\partial H}{\partial \mathbf{p}}\right)\right] \\
& +\boldsymbol{\nabla} \cdot[\frac{1}{4 \pi} \overbrace{\delta \Phi}^{\mathrm{E} 1}(\overbrace{\nabla \Phi}^{\mathrm{E} 1}+\frac{1}{c} \frac{\partial \mathbf{A}}{\partial t})]-\nabla \cdot[\frac{1}{4 \pi} \overbrace{\delta \mathbf{A}}^{\mathrm{M}} \times \overbrace{\mathbf{B}}^{\mathrm{M}}]
\end{aligned}
$$




$$
+\frac{1}{4 \pi c} \frac{\partial}{\partial t}[\overbrace{\delta \mathbf{A}}^{\mathrm{E} 2} \cdot(\overbrace{\nabla \Phi}^{\mathrm{E} 1}+\overbrace{\frac{1}{c} \frac{\partial \mathbf{A}}{\partial t}}^{\mathrm{E} 2})] \cdot
$$

\subsection{Local energy conservation}

The local energy conservation law is obtained by means of the usual Noether procedure. Contrary to [1], we consider here the usual shift variations and not the otherwise very convenient gauge-invariant shift variations because, besides gauge-invariant Lagrangians, we also want to investigate the non-gaugeinvariant case with $\mathcal{L}_{\mathrm{M}}=(1 / 8 \pi)\left(|\nabla \Phi|^{2}-\mathbf{B}^{2}\right)$.

Owing to a time shift $\epsilon_{t}$, the Euler variation of the total Lagrangian density is

$$
\delta \mathcal{L}_{\text {tot. }}(\mathbf{x}, t)=-\epsilon_{t} \frac{\partial}{\partial t} \mathcal{L}_{\text {tot. }}(\mathbf{x}, t) .
$$

For the actual development of the system, the kinetic Lagrange density $\mathcal{L}_{\mathrm{K}}(\mathbf{x}, t)$ does not contribute, because it vanishes after the Euler-Lagrange equations are inserted for $S$, i.e. the Hamilton-Jacobi equations, (48). One thus has

$$
\begin{aligned}
\delta \mathcal{L}_{\text {tot. }}(\mathbf{x}, t) & =\delta^{\mathrm{EL}} \mathcal{L}_{\text {tot. }}(\mathbf{x}, t)=\delta \mathcal{L}_{\mathrm{M}}(\mathbf{x}, t)=-\epsilon_{t} \frac{\partial}{\partial t} \mathcal{L}_{\mathrm{M}}(\mathbf{x}, t) \\
& =-\epsilon_{t} \frac{1}{8 \pi} \frac{\partial}{\partial t}[|\overbrace{\nabla}^{\mathrm{E} 1}+\overbrace{\frac{1}{c} \frac{\partial \mathbf{A}}{\partial t}}^{\mathrm{E} 2}|^{2}-\overbrace{\mathbf{B}^{2}}^{\mathrm{M}}],
\end{aligned}
$$

On the other hand, $\delta^{\mathrm{EL}} \mathcal{L}_{\text {tot. }}(\mathbf{x}, t)$ is also given by (50). Following a displacement $\epsilon_{t}$ in time, the usual shift variations of $\Phi, \mathbf{A}$ and $S$ are

$$
\delta \Phi=-\epsilon_{t} \frac{\partial \Phi}{\partial t}, \quad \delta \mathbf{A}=-\epsilon_{t} \frac{\partial \mathbf{A}}{\partial t}
$$

and

$$
\delta S=-\epsilon_{t} \frac{\partial S}{\partial t}=\epsilon_{t} H=\epsilon_{t}\left[e \Phi(\mathbf{x}, t)+\hat{H}\left(\mathbf{x}, \frac{\partial S}{\partial t}, t\right)\right],
$$

where (35) and (48) are used. By substituting these expressions in (50), with (45) and (46) taken into account, one obtains

$$
\begin{aligned}
& \frac{1}{\epsilon_{\mathrm{t}}} \delta^{\mathrm{EL}} \mathcal{L}_{\text {tot. }}(\mathbf{x}, t)=\underbrace{-\sum_{\text {p. s. }} \int d^{3} \alpha\left[\frac{\partial}{\partial t}\left(f_{\mathrm{p}} \hat{H}\right)+\frac{\partial}{\partial \mathbf{x}} \cdot\left(f_{\mathrm{p}} \hat{H} \frac{\partial \hat{H}}{\partial \mathbf{p}}\right)\right]}_{(a)}
\end{aligned}
$$

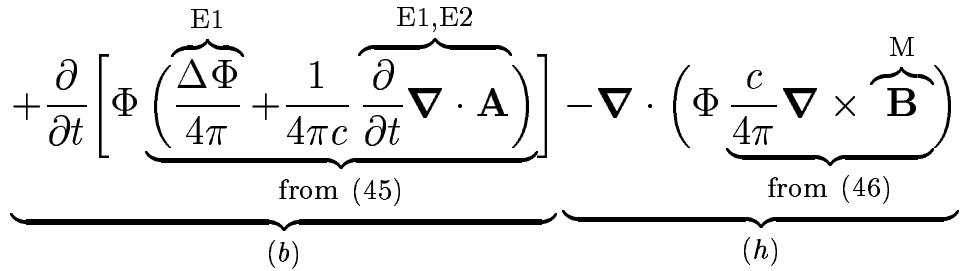




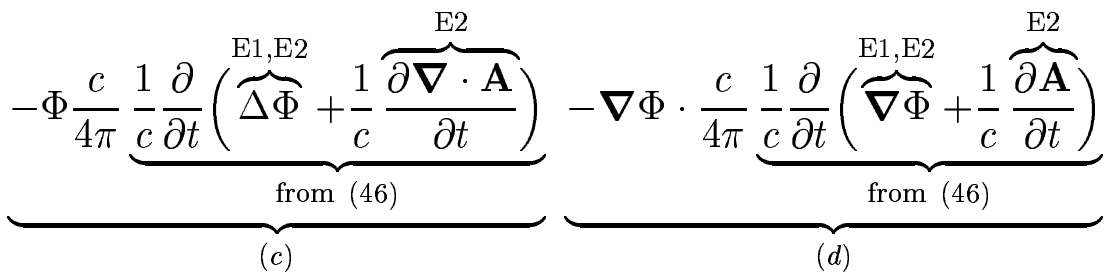

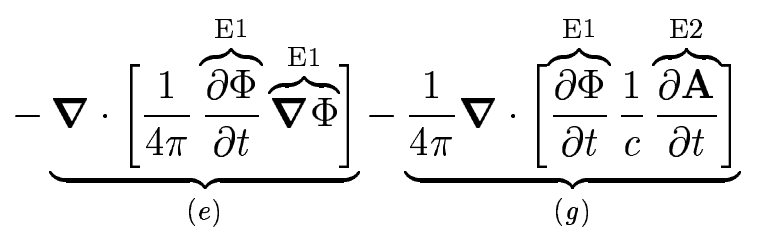

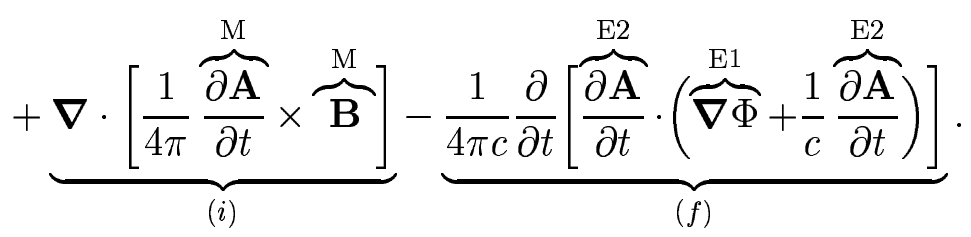

The term $(a)$ can be transformed by passing from the variable $\boldsymbol{\alpha}$ to $\mathbf{p}$, and from the primary distribution function $f_{\mathrm{p}}(\mathbf{x}, \boldsymbol{\alpha}, t)$ to the distribution function proper, $f(\mathbf{x}, \mathbf{p}, t)$, as explained in detail in [1], equations (5.22)-(5.30). In particular, this implies the substitution $\partial S(\mathbf{x}, \boldsymbol{\alpha}, t) / \partial \mathbf{x} \rightarrow \mathbf{p}$. One then obtains

$$
(a)=-\sum_{\text {p.s. }} \int d^{3} p\left[\frac{\partial}{\partial t}(f \hat{H}(\mathbf{x}, \mathbf{p}, t))+\frac{\partial}{\partial \mathbf{x}} \cdot\left(f \hat{H}(\mathbf{x}, \mathbf{p}, t) \frac{\partial \hat{H}}{\partial \mathbf{p}}\right)\right] .
$$

The term $(h)$ can be written as $+(c / 4 \pi) \nabla \cdot(\nabla \Phi \times \mathbf{B})$. Together with the term $(i)$, one obtains

$$
(h)+(i)=\boldsymbol{\nabla} \cdot\left[\frac{c}{4 \pi}(\boldsymbol{\nabla} \Phi+\frac{1}{c} \frac{\overbrace{\frac{\partial \mathbf{A}}{\partial t}}^{\mathrm{M}}}{\partial t} \times \overbrace{\mathbf{B}}^{\mathrm{M}}]=-\boldsymbol{\nabla} \cdot[\frac{c}{4 \pi} \mathbf{E} \times \overbrace{\mathbf{B}}^{\mathrm{M}}] .\right.
$$

Further, one finds

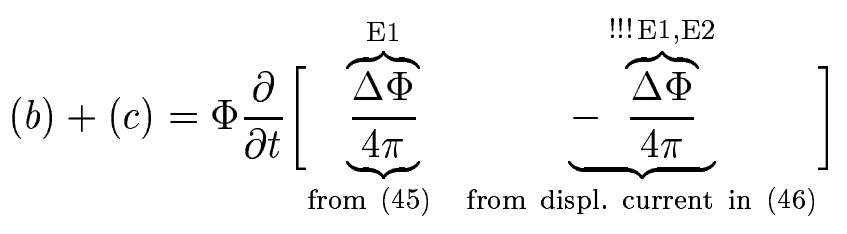

$$
\begin{aligned}
& +\frac{1}{4 \pi} \frac{\partial \Phi}{\partial t}[\underbrace{\boldsymbol{\nabla} \cdot \overbrace{\boldsymbol{\nabla} \Phi}^{\mathrm{E} 1}+\frac{\overbrace{1}^{\frac{\partial \boldsymbol{\nabla} \cdot \mathbf{A}}{c}}}{\partial t}}_{\text {from (45) }}]+\Phi \frac{1}{4 \pi c} \frac{\partial}{\partial t}[\overbrace{\text { from (45) }}^{\overbrace{\frac{\partial \boldsymbol{\nabla} \cdot \mathbf{A}}{\partial t}}^{\mathrm{E} 1, \mathrm{E} 2}}-\underbrace{\frac{\overbrace{\partial \boldsymbol{\nabla} \cdot \mathbf{A}}^{\mathrm{E} 2}}{\partial t}}_{\text {from }(46)}] \\
& =\Phi \frac{\partial}{\partial t}[\underbrace{\overbrace{\Delta \Phi}^{\mathrm{E} 1}}_{\text {from (45) }} \underbrace{-\overbrace{\Delta \Phi}^{\frac{\overbrace{\Delta \Phi}}{4 \pi}}}_{\text {from displ. current in (46) }}]
\end{aligned}
$$




$$
\begin{aligned}
& \underbrace{+\frac{1}{4 \pi} \boldsymbol{\nabla} \cdot[\frac{\partial \Phi}{\partial t} \overbrace{\boldsymbol{\nabla} \Phi}^{\mathrm{E}}]}_{A}-\underbrace{\frac{1}{4 \pi} \frac{\partial \boldsymbol{\nabla} \Phi}{\partial t} \cdot \overbrace{\boldsymbol{\nabla} \Phi}^{\mathrm{E} 1}}_{B}+\underbrace{\frac{1}{4 \pi} \frac{\partial \Phi}{\partial t} \frac{\overbrace{\frac{1}{c} \frac{\partial \boldsymbol{\nabla} \cdot \mathbf{A}}{\partial t}}^{\mathrm{E} 1, \mathrm{E} 2}}{\partial t}}_{\text {from (45) }} \\
& +\Phi \frac{1}{4 \pi c} \frac{\partial}{\partial t}[\overbrace{\text { from (45) }}^{\frac{\partial \boldsymbol{\nabla} \cdot \mathbf{A}}{\partial t}} \underbrace{\overbrace{\frac{\partial \boldsymbol{\nabla} \cdot \mathbf{A}}{\partial t}}^{\mathrm{E} 1, \mathrm{E} 2}}_{\text {from (46) }}],
\end{aligned}
$$

$$
\begin{aligned}
& (d)=\underbrace{-\frac{1}{4 \pi} \frac{\partial}{\partial t}[\boldsymbol{\nabla} \Phi \cdot \underbrace{(\overbrace{\boldsymbol{\nabla} \Phi}^{\mathrm{E} 1, \mathrm{E} 2}+\frac{\overbrace{1}^{\mathrm{E}} \frac{\partial \mathbf{A}}{\partial t})}{\mathrm{E} 2}]}_{\text {from displ. current in (46) }}}_{D} \\
& +\underbrace{\frac{1}{4 \pi} \frac{\partial \boldsymbol{\nabla} \Phi}{\partial t}}_{\text {factor of } B, E} \cdot \underbrace{\underbrace{\overbrace{\boldsymbol{\nabla} \Phi}^{\mathrm{E} 1, \mathrm{E} 2}}_{\text {factor of } B}+\underbrace{\overbrace{1}^{\frac{1}{c} \frac{\partial \mathbf{A}}{\partial t}}}_{\text {factor of } E})}_{\text {from displ. current in (46) }} \\
& (e)=\underbrace{-\frac{1}{4 \pi} \nabla \cdot[\frac{\partial \Phi}{\partial t} \overbrace{\nabla}^{\mathrm{E} 1} \overbrace{}^{\mathrm{\nabla} \Phi}]}_{A}, \\
& (g)=\underbrace{-\frac{1}{4 \pi} \frac{\overbrace{\frac{\partial \boldsymbol{\nabla} \Phi}{\partial t}}^{\mathrm{E} 1} \cdot \frac{1}{c} \frac{\partial \mathbf{A}}{\partial t}}{\mathrm{E} 2}}_{E} \underbrace{-\frac{1}{4 \pi} \overbrace{\frac{\partial \Phi}{\partial t} \frac{\overbrace{}^{2}}{c} \overbrace{\frac{\partial \boldsymbol{\nabla} \cdot \mathbf{A}}{\partial t}}^{\mathrm{E} 2}}^{\mathrm{E}}}_{C} .
\end{aligned}
$$

When all three contributions E1, E2 and M are present in the Maxwell part of the Lagrangian density, it is clear that the term $A$ of $(b)+(c)$ cancels the term $A$ of $(e)$, the term $B$ in $(b)+(c)$ cancels the same term in $(d)$, consisting of the two factors indicated in (59). The term $C$ in $(b)+(c)$ cancels the term $C$ in $(g)$, and the terms $E$ in $(d)$, consisting of the two factors indicated in (59), and $(g)$ cancel each other.

In cancelling $\Delta \Phi$, the term marked with !!! in $(b)+(c)$, Eq. (58), plays a crucial role, and the term $B$ in $(d)$, consisting of the two factors indicated in (59), is crucial to cancelling the same term in $(b)+(c)$. These terms are not present if the displacement current is not taken into account in the Euler-Lagrange equation for the current density, which is the case when $|\nabla \Phi|^{2} / 8 \pi$ instead of $|\boldsymbol{\nabla} \Phi+(1 / c) \partial \mathbf{A} / \partial t|^{2}$ is used in the Maxwellian part of the Lagrangian. 
The contribution $D$ from $(d)$, Eq. (59), and the term $(f)$, Eq. (55), finally yield

$$
\begin{aligned}
D+(f) & =-\frac{1}{4 \pi} \frac{\partial}{\partial t}[\boldsymbol{\nabla} \Phi \cdot(\underbrace{\overbrace{\boldsymbol{\nabla} \Phi}^{\mathrm{E} 1 \mathrm{E} 2}+\overbrace{\frac{1}{c} \frac{\partial \mathbf{A}}{\partial t}}^{\mathrm{E} 2}}_{\text {from displ. current in }})+\overbrace{\boldsymbol{\nabla} \Phi}^{\mathrm{E} 1} \cdot \overbrace{\frac{1}{c} \frac{\partial \mathbf{A}}{\partial t}}^{\mathrm{E} 2}+\overbrace{\left(\frac{1}{c} \frac{\partial \mathbf{A}}{\partial t}\right)^{2}}^{\mathrm{E} 2}] \\
& =-\overbrace{-\frac{1}{4 \pi} \frac{\partial \mathbf{E}^{2}}{\partial t}}^{\mathrm{E} 2}=-\overbrace{\frac{2}{8 \pi} \frac{\partial \mathbf{E}^{2}}{\partial t}}^{\mathrm{E} 2} .
\end{aligned}
$$

The local energy conservation law is then obtained in the usual way by setting $\delta^{\mathrm{EL}} \mathcal{L}_{\text {tot. }}(\mathbf{x}, t)$ from $(52)$ equal to $\delta^{\mathrm{EL}} \mathcal{L}_{\text {tot. }}(\mathbf{x}, t)$ from $(55)$, with $(56)$ - $(62)$ taken into account. In the following, four different forms of the Lagrangian density are considered.

\subsubsection{Gauge-invariant $\mathcal{L}_{\mathrm{M}}=(1 / 8 \pi)\left[|\boldsymbol{\nabla} \Phi+(1 / c) \partial \mathbf{A} / \partial t|^{2}-\mathbf{B}^{2}\right]$}

The result obtained with the exact Maxwell Lagrangian is, therefore,

$$
\frac{1}{\epsilon_{\mathrm{t}}} \delta^{\mathrm{EL}} \mathcal{L}_{\text {tot. }}(\mathbf{x}, t)=-\frac{\partial \varepsilon_{\text {kin. }}}{\partial t}-\boldsymbol{\nabla} \cdot \boldsymbol{\eta}_{\text {kin. }}-\frac{2}{8 \pi} \mathbf{E}^{2}-\boldsymbol{\nabla} \cdot\left[\frac{c}{4 \pi} \mathbf{E} \times \mathbf{B}\right],
$$

where

$$
\varepsilon_{\text {kin. }}=\sum_{\text {p.s. }} \int d^{3} p f \hat{H}
$$

and

$$
\boldsymbol{\eta}_{\text {kin. }}=\sum_{\text {p. s. }} \int d^{3} p f \hat{H} \frac{\partial \hat{H}}{\partial \mathbf{p}} .
$$

In this case one obtains the local energy conservation law

$$
\frac{\partial \varepsilon_{\text {kin. }}}{\partial t}+\nabla \cdot \boldsymbol{\eta}_{\text {kin. }}+\frac{\partial}{\partial t}\left[\frac{1}{8 \pi}\left(\mathbf{E}^{2}+\mathbf{B}^{2}\right)\right]+\nabla \cdot\left[\frac{c}{4 \pi} \mathbf{E} \times \mathbf{B}\right]=0
$$

It should be mentioned here as a side note that it is much easier to obtain this result by using the gauge-invariant shift variations introduced in [1]. As shown there, these yield

$$
\delta S=\epsilon_{t} \hat{H}, \quad \delta \Phi=0, \quad \delta \mathbf{A}=\epsilon_{t} c \mathbf{E},
$$

which, when inserted in (50), immediately yield (63). Of course, this is not of importance for the subject of the present paper.

\subsubsection{Non-gauge-invariant approximation with $\mathcal{L}_{\mathrm{M}}=(1 / 8 \pi)\left[|\boldsymbol{\nabla} \Phi|^{2}-\mathbf{B}^{2}\right]$}

This form of the Lagrangian density is sometimes used in the literature for the case of slowly-varying fluctuations. It is then said that the inductive part of the electric field can be neglected in the Lagrangian density, as compared with the 
contribution from the scalar gradient. A basic discussion of this argument is given in the Introduction. It will now be shown that this choice of Lagrangian leads to incorrect results, as concerns not only charge conservation but also, in particular, the local energy conservation law.

If the contribution $(1 / c) \partial \mathbf{A} / \partial t$ to the Maxwellian part $\mathcal{L}_{\mathrm{M}}$ of the Lagrangian is not taken into account, there are several consequences:

- The Euler-Lagrange equations (45) and (46) become equations (24), implying violation of local charge conservation.

- Since there is no displacement current in the equation for the current density, (46), there is, in particular, no term $(1 / 4 \pi) \partial \nabla \Phi / \partial t$, which would otherwise enter the local energy conservation law. Several terms containing $(1 / c) \partial \mathbf{A} / \partial t$ vanish. As a result, the term $-(2 / 8 \pi) \partial \mathbf{E}^{2} / \partial t$ disappears from (55), as follows from (62).

- In $(b)+(c),(58)$, the terms

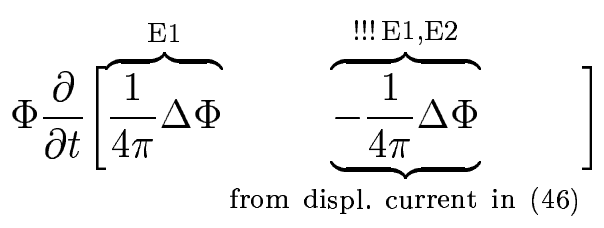

cannot cancel since the term $\overbrace{\ldots}^{\text {!! E1,E2 }}$ is discarded, and the term $B$,

$$
-\frac{1}{4 \pi} \frac{\partial \boldsymbol{\nabla} \Phi}{\partial t} \cdot \overbrace{\boldsymbol{\nabla} \Phi}^{\mathrm{E} 1}
$$

is not cancelled either since there is no corresponding term in $(d),(59)$.

For these now remaining terms one has

$$
\begin{aligned}
\Phi & \frac{\partial}{\partial t}\left[\frac{1}{4 \pi} \Delta \Phi\right]-\frac{1}{4 \pi} \frac{\partial \boldsymbol{\nabla} \Phi}{\partial t} \cdot \boldsymbol{\nabla} \Phi= \\
& \frac{1}{4 \pi} \boldsymbol{\nabla} \cdot\left[\Phi \frac{\partial \boldsymbol{\nabla} \Phi}{\partial t}\right]-\frac{1}{4 \pi} \boldsymbol{\nabla} \Phi \cdot \frac{\partial \boldsymbol{\nabla} \Phi}{\partial t}-\frac{1}{4 \pi} \frac{\partial \boldsymbol{\nabla} \Phi}{\partial t} \cdot \boldsymbol{\nabla} \Phi \\
= & \frac{1}{4 \pi} \boldsymbol{\nabla} \cdot\left[\Phi \frac{\partial \boldsymbol{\nabla} \Phi}{\partial t}\right]-\frac{1}{4 \pi} \frac{\partial|\boldsymbol{\nabla} \Phi|^{2}}{\partial t} .
\end{aligned}
$$

This introduces a wrong term into the local energy flux density, and also a term $-(1 / 4 \pi) \partial|\nabla \Phi|^{2} / \partial t$ which, in spite of having a form apparently correct for the local energy density, has not been correctly derived either.

In the present case one obtains in a way similar to that of the previous case the (incorrect) local conservation law

$$
\begin{aligned}
& \frac{\partial \varepsilon_{\text {kin. }}}{\partial t}+\boldsymbol{\nabla} \cdot \boldsymbol{\eta}_{\text {kin. }}+\frac{\partial}{\partial t}\left[\frac{1}{8 \pi}\left(|\boldsymbol{\nabla} \Phi|^{2}+\mathbf{B}^{2}\right)\right] \\
& +\boldsymbol{\nabla} \cdot\left[\frac{c}{4 \pi} \mathbf{E} \times \mathbf{B}\right]-\frac{1}{4 \pi} \boldsymbol{\nabla} \cdot\left[\Phi \frac{\partial \boldsymbol{\nabla} \Phi}{\partial t}\right]=0 .
\end{aligned}
$$


The spurious term $-(1 / 4 \pi)[\Phi(\partial \boldsymbol{\nabla} \Phi / \partial t)]$ in the energy flux density is also obtained within the framework of non-gauge-invariant drift-kinetic and gyrokinetic theories, e.g. as appears in Eq. (49) of [2], and Eq. (21) of [3], and is erroneously attributed to a current density wrongly identified as a polarization current density. It is, of course, not present in the gauge-invariant theories derived in [1], [4] and [5].

As already mentioned, the spurious terms obviously do not contribute if only the total energy of the system is considered, as done in, for example, [6]. There, the same non-gauge-invariant Maxwell Lagrangian density of this section is supplemented by a Lagrange multiplier term which formally guarantees local charge conservation. Although the expression for the current density remains incomplete and a different spurious term would appear in the local energy conservation law if this were derived, the spurious term is in this case related to the term $\partial \mathbf{A} / \partial t$ (and not to $\Phi(\partial \boldsymbol{\nabla} \Phi / \partial t)$, and is negligible according to the assumptions made in [6]; it is, therefore, of no consequence.

\subsubsection{Difference of neglecting $(1 / c) \partial \mathrm{A} / \partial t$ in $\mathcal{L}_{\mathrm{M}}$ or in final results}

It is easily seen that it is not equivalent to set

$$
\mathbf{E}=-\nabla \Phi-\frac{1}{c} \frac{\partial \mathbf{A}}{\partial t} \Rightarrow-\nabla \Phi
$$

right from the beginning in $\mathcal{L}_{\mathrm{M}}$, or in the final results in the local energy conservation law. Neglecting $(1 / c) \partial \mathbf{A} / \partial t$ in $\mathcal{L}_{\mathrm{M}}$ leads to the (wrong) result of (71). By neglecting it in the conservation law one obtains from (66) an expression different from (71), viz.

$$
\frac{\partial \varepsilon_{\text {kin. }}}{\partial t}+\boldsymbol{\nabla} \cdot \boldsymbol{\eta}_{\text {kin. }}+\frac{\partial}{\partial t}\left[\frac{1}{8 \pi}\left(|\boldsymbol{\nabla} \Phi|^{2}+\mathbf{B}^{2}\right)\right]+\boldsymbol{\nabla} \cdot\left[\frac{c}{4 \pi} \mathbf{E} \times \mathbf{B}\right]=0,
$$

which is the correct expression for the local energy conservation law for the approximation $|(1 / c)(\partial \mathbf{A} / \partial t)| \ll|\nabla \Phi|$. Note that the contribution from the vector potential is kept in the Poynting vector since only that contribution determines $\boldsymbol{\nabla} \times \mathbf{E}=-(1 / c)(\partial \mathbf{B})(\partial t)$.

\subsubsection{Gauge-invariant approximation with $\mathcal{L}_{\mathrm{M}}=(1 / 8 \pi)\left[|\boldsymbol{\nabla} \Phi+(1 / c) \partial \mathbf{A} / \partial t|^{2}\right]$}

This approximation can be useful for describing of electrostatic perturbations in the context of linearized theory. In order to stay within the above investigation we do that here for the nonlinear theory. This exhibits the same kind of features as can be found with the linearized theory. Basically, the Maxwell Lagrangian density for an electrostatic theory is given by

$$
\mathcal{L}_{\mathrm{M}}=\frac{1}{8 \pi}\left[\boldsymbol{\nabla} \Phi+\frac{1}{c} \frac{\partial \mathbf{A}}{\partial t}\right]^{2}=\frac{1}{8 \pi} \mathbf{E}^{2} .
$$


The contributions $\overbrace{*}^{\mathrm{M}}$ in $(33)$ and following equations vanish. The EulerLagrange equations (45) and (46) therefore become

$$
\begin{gathered}
\frac{1}{4 \pi} \boldsymbol{\nabla} \cdot \mathbf{E}=\sum_{\mathrm{p} . \mathbf{s} .} e \int d^{3} \alpha f_{\mathrm{p}}(\mathbf{x}, \boldsymbol{\alpha}, t)=\rho \\
\frac{1}{4 \pi} \frac{\partial \mathbf{E}}{\partial t}+\mathbf{j}=\frac{1}{4 \pi} \frac{\partial \mathbf{E}}{\partial t}+\sum_{\text {p. s. }} e \int d^{3} \alpha f_{\mathrm{p}}(\mathbf{x}, \boldsymbol{\alpha}, t) \frac{\partial H}{\partial t \mathbf{p}}=0 .
\end{gathered}
$$

The local energy conservation law obtained from (52) and (55)-(62) becomes

$$
\frac{\partial \varepsilon_{\text {kin. }}}{\partial t}+\nabla \cdot \boldsymbol{\eta}_{\text {kin. }}+\frac{1}{8 \pi} \frac{\partial}{\partial t} \mathbf{E}^{2}=0
$$

There is no magnetic field energy and no Poynting flux.

\subsubsection{Gauge-invariant approximation with $\mathcal{L}_{\mathrm{M}}=-(1 / 8 \pi) \mathbf{B}^{2}$}

In an approximation in which a plasma is considered as quasi-neutral, with displacement current neglected, the Maxwell part of the Lagrange density is basically given by

$$
\mathcal{L}_{\mathrm{M}}=-\frac{1}{8 \pi} \mathbf{B}^{2}
$$

One still has, however,

$$
\mathbf{E}=-\nabla \Phi-\frac{1}{c} \frac{\partial \mathbf{A}}{\partial t}
$$

The contributions $\ldots$ E1, E2 in (33) and following equations vanish. The Euler-Lagrange equations (45) and (46) in this case become

$$
\begin{gathered}
0=\rho=\sum_{\text {p. s. }} e \int d^{3} \alpha f_{\mathrm{p}}(\mathbf{x}, \boldsymbol{\alpha}, t), \\
\frac{c}{4 \pi} \boldsymbol{\nabla} \times \mathbf{B}=\mathbf{j}=\sum_{\text {p. s. }} e \int d^{3} \alpha f_{\mathrm{p}}(\mathbf{x}, \boldsymbol{\alpha}, t) \frac{\partial H}{\partial \mathbf{p}} \rightarrow \boldsymbol{\nabla} \cdot \mathbf{j}=0 .
\end{gathered}
$$

The local energy conservation law obtained from (52) and (55)-(62) becomes

$$
\frac{\partial \varepsilon_{\text {kin. }}}{\partial t}+\boldsymbol{\nabla} \cdot \boldsymbol{\eta}_{\text {kin. }}+\frac{1}{8 \pi} \frac{\partial}{\partial t} \mathbf{B}^{2}+\frac{c}{4 \pi} \boldsymbol{\nabla} \cdot(\mathbf{E} \times \mathbf{B})=0
$$

There is no electric field energy in this case.

\section{Summary}

It has been shown that the use of special gauges in the action integral for theories involving electromagnetic fields makes it necessary to supplement Hamilton's principle by a Lagrange multipliers formalism which guarantees the validity of these gauges. Examples considered are the Coulomb and the Lorentz 
gauges and the gauge $\Phi=0$, with the result that the Euler-Lagrange equations in each case are the exact Maxwell equations together with the respective conservation laws.

Consistent sets of Euler-Lagrange equations also result when gauge-invariant approximations, e.g. the quasi-neutral or the electrostatic linearized theories are used in the action integral, with corresponding meaningful local conservation laws (only the local energy conservation law is considered).

Non-gauge-invariant approximations, however, lead to inconsistent sets of EulerLagrange equations. A detailed investigation of an example of such an approximation, which is used in the literature, shows that certain supposedly obviously reasonable approximations are by no means reasonable. The deficiencies of the non-gauge-invariant approximations result in wrong terms in the local energy conservation law. Although these terms do not influence the total energy conservation, they describe a wrong underlying dynamics.

In the present paper, only investigations concerning the Euler-Lagrange equations and the local energy conservation law were of interest. The aim was to compare different theories characterized by different Maxwell Lagrangian densities. An important result was that it is not equivalent to neglect $(1 / c) \partial \mathbf{A} / \partial t$ in the Maxwell Lagrangian density or only in the final results obtained with Hamilton's principle. This is related to the fact that within the variational problem one does not compare the contributions of the factors of $\delta \Phi$ with the contributions of the factors of $\delta \mathbf{A}$. In fact, the comparison is made separately within the factor of $\delta \Phi$ and within the factor of $\delta \mathbf{A}$. Results concerning the derivation of a symmetric energy-momentum tensor for gauge-invariant driftkinetic and gyrokinetic theories are found in [1], [4] and [5].

\section{References}

[1] Correa-Restrepo, D. and Pfirsch, D. 2004 Noether formalism with gaugeinvariant variations. J. Plasma Phys. 70, 199-213.

[2] Brizard, A. 2000 Variational principle for nonlinear gyrokinetic MaxwellVlasov equations. Phys. Plasmas 7, 4816-4822.

[3] Brizard, A. J. 2000 New Variational Principle for the Vlasov-Maxwell Equations. Phys. Rev. Lett. 84, 5768-5771.

[4] Pfirsch, D. and Correa-Restrepo, D. 2004 New method of deriving local energy and momentum-conserving Maxwell-collisionless drift-kinetic and gyrokinetic theories: basic theory. Accepted for publication, J. of Plasma Phys. 70, part 6 .

[5] Correa-Restrepo, D. and Pfirsch, D. 2004 New method of deriving local energy and momentum-conserving Maxwell-collisionless drift-kinetic and 
gyrokinetic theories: conservation laws and their structures. Accepted for publication, J. of Plasma Phys. 70, part 6.

[6] Sugama, H. 2000 Gyrokinetic field theory. Phys. Plasmas 7, 466-480. 